\title{
À la claire Fontaine : un poète gaélique au Maghreb
}

'À la Claire Fontaine': a Gaelic poet in the Maghreb

\section{Michel Byrne}

\section{OpenEdition}

\section{Journals}

Édition électronique

URL : https://journals.openedition.org/lbl/296

DOl $: 10.4000 / / b l .296$

ISSN : 2727-9383

\section{Éditeur}

Université de Bretagne Occidentale - UBO

\section{Édition imprimée}

Date de publication : 1 mai 2017

Pagination : 209-238

ISBN : 979-10-92331-31-8

ISSN : $1270-2412$

\section{Référence électronique}

Michel Byrne, «À la claire Fontaine : un poète gaélique au Maghreb », La Bretagne Linguistique [En ligne], 21 | 2017, mis en ligne le 01 mai 2020, consulté le 29 mars 2022. URL : http:// journals.openedition.org//bl/296; DOI : https://doi.org/10.4000/lbl.296

\section{(c) (1)}

La Bretagne Linguistique est mise à disposition selon les termes de la Licence Creative Commons Attribution 4.0 International. 


\section{À la claire Fontaine : un poète gaélique au Maghreb}

En 1940 le grand poète moderniste Hugh MacDiarmid - tête de file depuis les années vingt du mouvement que le philosophe Denis Saurat dénomme la Renaissance Écossaise - sort son anthologie historique de la poésie écossaise, The Golden Treasury of Scottish Poetry. Pour la première fois se côtoient sur la page poèmes anglais, scots, latins et gaéliques (ces deux derniers courants représentés en versions anglophones). Luimême non-gaélophone mais très conscient que son public connaît mal l'héritage gaélique, MacDiarmid a tenu à inclure dans son recueil de grandes œuvres des siècles passés et un écrivain du XXe siècle, et il annonce de surcroît l'arrivée sur scène de deux jeunes poètes "très remarquables ${ }^{1} »$ : Somhairle MacGill-Eain (Sorley Maclean, 19111997) qui deviendra en effet l'incontournable du modernisme gaélique et sera considéré l'égal de MacDiarmid; et George Campbell Hay (1915-1984), moins iconoclaste que son aîné et dans un premier temps moins original, mais qui incarne déjà tous les principes phares de la Renaissance, à la fois par son trilinguisme littéraire (gaélique, scots et anglais) et par son ouverture aux langues et aux cultures européennes (il produit des traductions convaincantes de poèmes irlandais, scandinaves et grecs) : le rejet de l'anglocentrisme culturel et politique et

* Maitre de conférences de gaélique et celtique à l'université de Glasgow (Oilthigh Ghlaschu)

1. H. MACDiARmid, The golden treasury of Scottish poetry, (London, Macmillan, 1940) Edinburgh, Canongate, 1993, p. 351. 
du provincialisme ; le renouvellement de passerelles entre l'Écosse et l'Europe ; la revalorisation du parler écossais (scots) et de sa littérature ; et la réintégration du gaélique au cœur de la vie culturelle du pays ${ }^{2}$. Hay est le premier poète écossais - et restera longtemps le seul - à écrire dans les trois langues autochtones ${ }^{3}$, mais il est aussi l'unique écrivain gaélophone (jusqu'aux années quatre-vingt) à s'adonner de façon soutenue à la traduction poétique ${ }^{4}$.

À cette époque son travail est marqué surtout par un éclectisme linguistique et littéraire, et des capacités très fortes dans la technique poétique. Ses thématiques ne sont pas neuves (il est surtout poète de la mer et des pêcheurs du littoral de l'ouest, et poète intimiste de la nature), mais pendant ses années de service dans l'armée britannique sa voix gagnera en originalité : il deviendra un éloquent poète de guerre et un poète philosophe, et les thèmes maghrébins qu'il aborde apporteront un élément très nouveau à la littérature gaélique. Cet article propose d'examiner les influences francophones sur son travail durant la guerre.

\section{Biographie du poète}

Né en 1915, George Campbell Hay passe sa jeune enfance dans le petit port de pêche de Tarbert Loch Fyne, à l'extrémité nord de la péninsule de Kintyre (du gaélique Cinn Tìre : "penn-tir», Finisterre), dans l'Argyllshire, berceau historique de la culture gaélique en

2. J. Derrick MaCCluRE, Language, poetry and nationhood, East Linton, Tuckwell, 2000 ; en particulier chapitre 7 : «The MacDiarmid Revolution ». Voir aussi Margery Palmer MCCulLOCH, Scottish Modernism and its contexts 1918-1959, Edinburg, EUP, 2009, p. 95101 ("Celtic connections »); et B. SELLIN (dir.), L'Écosse, Cahier de l'Institut culturel de Bretagne, $n^{\circ}$ 6, Brest, Skol-Uhel ar Vro, 1986, p. 98-100. Pour une discussion des influences racistes européennes sur la « Gaelic Idea » de MacDiarmid voir Keith DiXON, «Hugh MacDiarmid and the Gaelic Idea », Cahiers de Bretagne Occidentale 7 : Pays de Galles, Écosse, Irlande, Brest, CRBC, 1987, p. 169-184.

3. Par la suite, seuls deux poètes manifesteront un tel trilinguisme, à divers degrés : William Neill (1922-2010) locuteur scots de l'Ayrshire, et l'éminent poète gaélique Aonghas MacNeacail (1944-), de l'île de Skye.

4. La pratique était pourtant répandue parmi les poètes écossais anglo-scotophones; voir J. Derrick MCCLuRE, " George Campbell Hay's translations from Italian poetry », in S. Arbuthnot, K. Hollo (dir.), Fil Súil $n$ Glais - A grey eye looks back, Ceann Drochaid, Clann Tuirc, 2007, p. 87-96, à 87. Aussi P. FrANCE, D. GLEN (dir.), European poetry in Scotland, Edinburgh, EUP, 1989. 
Écosse. Région fortement gaélophone, le scots et l'anglais y prennent pied dès le XVIII ${ }^{\mathrm{e}}$ siècle, se propageant par l'industrie de la pêche et les migrations saisonnières. Quand les Hay (scotophones de l'Ayrshire) s'installent à Tarbert dans les années 1830, ils acquièrent vite la langue courante du village, mais ce plurilinguisme ne durera qu'à peine deux générations. Le père du poète, le pasteur et romancier John MacDougall Hay (1879-1919), est uniquement anglo-scotophone ${ }^{5}$. La mère du poète, Catherine Campbell, de famille hébridéenne, n’hérite pas non plus de la langue de ses parents. Rappelons aussi que la scolarité nationale monolingue établie en 1872 a sonné le glas des écoles gaéliques ${ }^{6}$. Sans statut légal ni prestige dans le pays, la pratique de la langue diminue dans toute la région des Highlands, une érosion encore plus marquée dans les contrées limitrophes du sud, tel que l'Argyllshire. Parmi les locuteurs, l'analphabétisme deviendra la norme jusqu'à la seconde moitié du $\mathrm{XX}^{\mathrm{e}}$ siècle.

Au tournant du siècle, on ne compte à Tarbert qu'une dizaine de gaélophones monolingues et environ $45 \%$ de bilingues, et après la première guerre, la langue y est bel et bien en voie d'extinction (cependant elle influe manifestement sur le lexique et la syntaxe du parler scots majoritaire). Enfant, George Hay n'entendra la langue que parmi les personnes âgées comme les pêcheurs qu'il fréquente jeune, suite au décès de son père. Dans la maison avoisinante, ses grands-tantes ne pratiquent quasiment jamais leur langue maternelle, devenue pour elles comme pour beaucoup d'autres locuteurs de cette génération une « langue stigmate », dépassée et retardataire ${ }^{7}$. Il prend néanmoins conscience très jeune du soubassement celtique de son environnement et cultive la langue par l'écoute et par la lecture assidue de tout ce qu'il trouve à portée de main (en l'occurrence, écritures saintes, sermons et recueils de poésie). Sa double appartenance culturelle restera au cœur de son identité et de sa conception de l'Écosse, comme il l'exprimera

5. Angus MarTin, Kintyre the bidden past, Edinburgh, John Donald, 1999, p. 26-47 : «The Gaelic decline ».

6. Charles W. J. WitheRs, Gaelic Scotland : the transformation of a culture region, London, Routledge, 1988, p. 313 et 163.

7. Francis FAVEREAU, Bretagne contemporaine : langue, culture, identité, Morlaix-Montroulez, Skol Vreizh, 1993, p. 128, à propos de «l'identité négative » du breton à la même époque. Pour l'information biographique, MARTIN, op. cit., p. 34 et 53. 
en 1940 en conclusion du poème lyrique "Ceithir gaothan na h-Alba » («Les quatre vents de l'Écosse ») :

"Fad na bliadhna, rè gach raìthe, gach là 's gach ciaradh feasgair dhomb 'S i Alba nan Gall's nan Gaidheal is gaire, is blàths, is beatha dhomb ${ }^{8}$." « Toute l'année, à chaque saison, chaque jour et à chaque tombée de la nuit

L'Écosse des Lowlanders et des Gaëls est mon allégresse, ma chaleur, ma vie ${ }^{9}$.»

Cette déclaration (taillée à présent dans la paroi du parlement écossais) peut sembler d'un bon sentiment irréprochable et même anodin, mais à l'époque la revendication d'une égalité culturelle entre Basses et Hautes-Terres comportait une part de déf ${ }^{10}$. Dans un premier temps le poète aimera aussi fixer des titres gaéliques à ses poèmes anglo-scotophones traitant de Kintyre, comme pour attiser la mémoire collective et rappeler qu'il y a eu usurpation culturelle; ainsi Tì Thäirngire (Terre promise), Combradh nan rubba (Le dialogue des pointes), Lomsgrios na tire (La dévastation du pays).

La sensibilité linguistique très éveillée de Hay trouvera un appoint important dans sa scolarité. En tant que fils de pasteur décédé, on lui octroie à dix ans une bourse pour les écoles élitistes d'Édimbourg. Envoyé en pension, il finira par suivre un cursus d'études classiques à Oxford, bien que son penchant d'adolescent soit bien plus fort pour les langues celtiques et scandinaves ${ }^{11}$. Hormis ces années d'études universitaires et son service de guerre, il passera le restant de sa vie à Édimbourg, loin de sa terre natale. Son parcours scolaire en littérature et en études humanistes cultive en lui un sentiment européen très fort, qui facilitera son rejet de l'identité britannique et l'aide à développer une facilité étonnante de mouvance linguistique et littéraire, entre langues, registres et traditions. C'est cette sensibilité qui lui permettra de s'épanouir et de trouver sa propre voie - sa propre voix - quand il atteindra le Maghreb.

\footnotetext{
8. G. C. HAY, Collected Poems and Songs, Edinburgh, EUP, 2003, p. 215.

9. Les traductions du gaélique sont de l'auteur de cet article.

10. A. Dosa \& M. MACLEOD, «Contemporary poetry (1950-) », dans C. Sassi (dir.), The International Companion to Scottish Poetry, Glasgow, SLI, p. 83-93, et 85.
}

11. MARTIN, op. cit., p. 64-65. 
Son parcours éducatif dans un milieu haut-bourgeois anglais aura peut-être intensifié un sentiment d'altérité à l'adolescence. Hay revient d'Oxford en 1938, patriote acharné (de gauche), et cet engagement s'entend fortement dans les poésies de cette période. C'est ce patriotisme écossais et sa haine de l'impérialisme britannique qui le pousseront à refuser la conscription militaire en 1940. Il n'est pas seul : une petite minorité de radicaux républicains, socialistes et indépendantistes revendiquent le droit de l'Écosse à la neutralité, à l'instar de l'Irlande, se disant prêts à défendre le pays en cas d'attaque mais non à porter les armes pour l'Empire. Hay s'exprimera en scots dans une « vulgaire ballade » restée inédite :

"They thocht tie herd us like a wheen o shocblin hairy yowes,

I think they'll find us thrawn eneuch, an' ragged fowk tie rowse;

Their cratur King an' me, ma boys, we little do agree,

I'll no come rinnin like a tyke though he should whustle me ${ }^{12}$."

« Ils ont voulu nous rassembler tels des brebis poilues peinardes,

Mais ils nous trouveront entêtés et bourrus lorsque provoqués ;

Leur p'tit bonhomme de Roi et moi, les gars, on ne s'entend guère,

Et s'il me siffle, je ne vais pas accourir comme un clébard. »

On ne trouvera pas la même dérive fascisante dans le mouvement neutraliste/anti-conscriptionniste que dans certains milieux nationalistes bretons de l'époque, mais les remarques de Jean-Jacques Monnier sur la collaboration chez « les peuples non reconnus ou dominés » ne sont pas sans pertinence vis-à-vis de l'attitude équivoque de Hay : «Le ressentiment accumulé contre l'État était tel qu'il a freiné la prise de conscience par certains de la vraie nature du nazisme ${ }^{13}$. » Même parmi les nationalistes, la prise de position neutraliste en Écosse a très peu d'adhérents ${ }^{14}$, mais Hay persiste et est arrêté en 1941, après huit mois en fuite dans les montagnes d'Argyll. Envoyé en camp d'entraînement

12. "A vulgar ballad for the times », lettre de février 1940. Voir HAY, op. cit., p. 63 et 564.

13. Préface dans Kristian Hamon, Les nationalistes bretons sous l'occupation, Ar RelegKerhuon, An Here, 2001, p. 9.

14. L'anti-conscriptionnisme est brièvement adopté par le jeune Scottish National Party en 1937, mais vite révoqué. R. J. FINLAY, Independent and Free, Edinburgh, John Donald, 1994, p. 243. Voir aussi G. BowD, Fascist Scotland: Caledonia and the far right, Edinburgh, Birlinn, 2013. 
(où il retrouve son compère, le poète Sorley Maclean, lui-même tiraillé entre son horreur du fascisme et ses convictions anti-impérialistes), il écrit sur un ton décontenancé : "J'avais pourtant juré que je ne les laisserais pas avoir le dessus, mais me voilà en kaki. Mais les Allemands sont véritablement infects dans leur démarche, et il est peut-être juste d'être en kaki. D’un autre côté les Anglais ont été tout aussi néfastes, même récemment ${ }^{15}$. » Si l'on peut s'interroger sur les tensions suscitées par une position aussi équivoque, surtout relativement à la crise psychologique subie par le poète en 1946, on peut avancer avec un peu plus d'assurance que la position morale non ambiguë - peu ou prou pacifiste - élaborée par Hay dans sa poésie de guerre n’est pas sans être liée à son «schweikisme» au sein de l'armée britannique.

Affecté au service des magasins militaires, il prend part au débarquement allié à Alger en novembre 42 et suivra la $1^{\text {re }}$ armée britannique durant la campagne de Tunisie. Après la défaite des troupes de l'Axe en mai 43, il est chargé d'initier les prisonniers italiens « aux chinoiseries du service des magasins ${ }^{16}$ ", et il sert aussi d'interprète en français, italien et arabe, langues qu'il aura acquises avec une rapidité étonnante. Après la chute de Rome en juin 44, il continue son travail d'interprète et d'instructeur en Italie, où il complétera ses grands poèmes à thèmes arabes ; puis, muté au service de l'enseignement, il sera envoyé en Grèce au début 46. C'est à cette période, de 1944 à 1947, qu'il produira la plus grande partie de son œuvre. Mais dans le guêpier qu'est la Macédoine en 1946, se frayer parmi les gens du pays comporte des risques pour tout soldat britannique, même s'il parle aisément le grec ; en juin 46 Hay est victime d'une agression par une milice droitiste ${ }^{17}$ et est renvoyé en Écosse, profondément traumatisé. À Tarbert, il continue d'écrire et il prépare la publication de ses poèmes, mais après un effondrement

15. «I damned well said I wouldn't let them get the better of me. But here I am in khaki. Yet the Germans are a very pestilence in their doings and perhaps one should be in khaki. On the other hand the English have been just as bad even recently... Lettre de novembre 1941, HAY, op. cit., p. 472.

16. «Initiating Italians into the chinoiseries of the RAOC», lettre du 2 février 1944, National Library of Scotland (NLS) Acc. 6419/38a (NLS Acc. 6419/38a : lettres de Hay à son ami Douglas Young, poète, professeur de lettres classiques et à cette époque président du Scottish National Party, emprisonné pour sa démarche anti-conscriptionniste.)

17. Selon le souvenir du poète trente ans plus tard. Voir MARTIN, op. cit., p. 68. 
mental en 48 il est interné à l'hôpital psychiatrique d'Édimbourg. Il y restera douze ans, mais avant que sa voix ne s'éteigne, il sort trois recueils : Wind on Loch Fyne (Vent sur le Lac Fyne, 1948), où le scots et l'anglais font part égale (avec aussi une poignée de poèmes français composés au Maghreb) ; et deux ouvrages gaéliques : Fuaran Slèibh (Source de montagne, 1948), et $O$ na Ceithir Airdean (Des quatre vents, 1952). Avec le poème épique dont on ignorera l'existence pendant presque quarante ans, cette centaine de poèmes constitue son œuvre majeure.

Hay retrouve la liberté en 1960, mais une liberté qui sera cernée de traitements et de périodes d'alcoolisme. À partir des années soixante jusqu'à son décès en 1984 il écrira encore plus de deux cents poèmes, en gaélique surtout $(70 \%)$ et en scots $(25 \%)$. Grâce à des anthologies de marque dans les années soixante-dix ${ }^{18}$, il n'est pas tout à fait oublié, mais pendant que son contemporain Somhairle MacGill-Eain (Sorley Maclean) est de plus en plus loué par le monde littéraire anglophone, Hay reste caché du public. Néanmoins, il fait la une en 1983 quand sa plus grande œuvre des années de guerre, le long poème inédit et inachevé « Mochtàr is Dùghall » (Mokhtar et Dou'al), est découverte et obtient la palme littéraire de l'association gaélique nationale. Il meurt seul quelques mois plus tard, à l'âge de 69 ans.

Peu connaîtront l'étendue de son œuvre, jusqu'à ce que paraisse, plus de quinze ans après sa mort, l'édition complète de ses poèmes ${ }^{19}$. Si elle fait mieux comprendre l'envergure linguistique et thématique de ses écrits, l'édition posthume élude une part importante de son métissage culturel, à savoir les presque deux cents poèmes traduits de littératures étrangères et celtiques ${ }^{20}$ vers le gaélique, le scots ou l'anglais. Son recueil anglo-scotophone de 1948 et la collection gaélique de 1952 comptaient pourtant chacun une trentaine de textes, donc $50 \%$ de leur contenu, traduits de l'italien, du grec ou de l'arabe. Parmi ses traductions les plus réussies on compte des sonnets de Pétrarque, dans un

\footnotetext{
18. Noter en particulier D. MaCAmHLAigh (dir.), Nua-bhàrdachd Ghäidblig | Modern Scottish Gaelic Poems, Edinburgh, Southside, 1976 ; Canongate, 1999, texte canonique de la poésie moderniste gaélique.

19. HAY, op. cit.

20. L'irlandais surtout, et quelques textes gallois. Hay n'a eu aucun contact avec le breton.
} 
gaélique souple et mesuré, et les sonnets railleurs de Cecco Angiolieri (XVe siècle) transposés avec beaucoup de bonheur en scots ${ }^{21}$.

\section{Un modernisme prudent : l'exemple du lamento (tuireadh)}

Si le modernisme de son ainé, Sorley Maclean (Somhairle MacGillEain, 1911-1996) est fulgurant dès ses débuts et constitue bien « une rompure délibérée avec la tradition ${ }^{22} »$, le cheminement de Hay est moins iconoclaste et plus tâtonnant. On situera l'originalité de sa poésie anglo-scotophone dans le courant gaélique qu'il infuse dans la rythmique et l'assonance. Dans ses poésies gaéliques, il tourne le dos à la tendance nostalgique et sentimentale de la poésie contemporaine et cherche à se détacher des conventions chansonnières du XIX siècle devenues rengaines et entravantes, non pas en allant dans la direction du vers libre, mais en cultivant des adaptations modernes de métriques anciennes ${ }^{23}$.

Pour illustrer sa démarche circonspecte, on peut citer sa réutilisation en 1940 du tuireadh, la lamentation quasiment païenne apparentée au caoineadh («keen ») irlandais, forme populaire inutilisée depuis plus de cent cinquante ans (quand la pratique du chant funèbre et des pleureuses tomba en désuétude dans les Highlands), mais dont des exemples sont préservés dans les chants de foulage hébridéens et dans des recueils imprimés. "Grunnd na mara » ("Le fond de l'océan ») de Hay est le cri de douleur d'une mère pour son fils noyé, et le poème ouvre par la bribe d'un véritable lamento du XVIII siècle, avant d'élaborer ses motifs intimes de perte et de deuil. Ayant calqué avec beaucoup d'adresse un mode d'expression traditionnel, Hay ajoutera au macabre du poème en faisant parler le noyé. Celui-ci reproche à sa mère de

21. On rappellera la remarque d'Henri Gibault sur « le lexique de dénigration » du scots : «La langue écossaise [...] détient tout un arsenal péjoratif infiniment plus meurtrier que celui que nous offre l'anglais. » H. GIBAULT, "Introduction », dans H. Gibault (dir.), Écosse Littérature et Civilisation, Grenoble, Université des langues et lettres de Grenoble, 1979, p. 7.

22. Christopher WHYTE, «Cultural Catalysts : Sorley Maclean and George Campbell Hay », dans Ian BROwN (dir.), Edinburgh History of Scottish Literature, vol. 3, Edinburgh, EUP, 2011, p. 62-151, et p. 153.

23. C'est dans la revue du leader moderniste Hugh MacDiarmid Voice of Scotland que Hay publie son «manifeste » en 1939. 
lui dérober son repos par ses pleurs incessants (un motif du folklore pêcheur trouvé également en Bretagne ${ }^{24}$ ), mais dénonce aussi l'auteur de sa mort - la métropole impériale : «Lunnainn a mharbh mi, | a mbill an t-sùil nach fhaca $i$ » («C'est Londres qui m'a tué, | qui a détruit le visage qu'elle ne vit jamais »). Le noyé présumé pêcheur est donc de la marine anglaise et le poète se prononce dans un dernier distique : «Mo losgadh, muinntir nan eilean - | Is daor a phäigh sibh mòrachd Bhreatainn 25 ! " ( La douleur me brûle, peuple des îles - | Vous l'avez payée cher, la gloire britannique!»).

On voit donc le poète reprendre une tradition désuète et la relancer à des fins contemporaines : ici, se servir du cri lyrique funèbre pour remettre en question l'engagement militaire écossais (ou gaélique) dans l'entreprise impérialiste britannique et, plus spécifiquement en 1940, contester les décrets conscriptionnistes de Londres. «Le fond de l'océan » reste très proche de ses sources littéraires, mais Hay pressentait dès 1939 que la métrique peu ornée du tuireadh (en paragraphes monorimes) se prêterait aisément à des applications plus variées, par exemple au mode narratif. C'est au Maghreb, cinq ans plus tard, que l'inspiration le relancera sur cette voie, quand il concevra son œuvre épique «Mochtàr is Dùghall » ("Mokhtar et Dou'al»). Cette grande fresque poétique ouvre (et se clôt) sur un tableau macabre : les cadavres pitoyables du tirailleur arabe et du fantassin Highlander atteints par un même obus sur les pentes du Djebel Zaghouan (dernier site de résistance des troupes de l'Axe en Tunisie). Si la société gaélique a depuis longtemps délaissé le lamento, au Maghreb la tradition des pleureuses est encore bien vivante en dehors des villes, et avant de procéder aux récits des aïeux qui feront le gros de l'œuvre, Hay ressuscite une deuxième fois la métrique du tuireadh pour donner la parole à la jeune veuve arabe. Ainsi le Maghreb rencontre les Hautes-Terres de l'Écosse dans un monologue qui évoque le rang subalterne des deux cultures en fusionnant à la fois locutions et tournures arabes :

24. Voir A. Le Braz, La légende de la mort chez les Bretons armoricains, Paris, H. Champion, 1928, p. 100-105: «Il ne faut point trop pleurer l'anaon.»

25. La fierté impériale était loin d'être rare parmi les Gaéls du XIX ${ }^{\mathrm{e}}$ siècle et figurait notamment dans les éloges militaires; encore au début du $\mathrm{XX}^{\mathrm{e}}$ siècle un long poème sur « la grandeur britannique », « Mòrachd Bhreatainn », par un écrivain réputé, gagnait la palme littéraire. 
«Écoutez toutes, femmes du douar! | Les armées des Roumis se sont réunies | dans la vendetta des souverains et des territoires. | Ils ont fait parler la poudre | dans les montagnes orientales du pays de Tunis»;

l'adaptation d'une réelle lamentation arabe :

«Le foyer de mon cœur est tout embrasé | pour mon époux, soutient de ma demeure. | ... Dans sa demeure on oubliera | qu'il revenait chaque soir. | ... Ne placez pas votre confiance en ce monde - | il est impitoyable et sinistre trompeur ${ }^{26} ! »$;

et la fureur outrée de certaines lamentations gaéliques appelant à la vengeance :

«Ô mes frères et vous, frères de mon père! | Lisez les traces et cherchez dans le vent le courant | de l'homme qui l'abattit sans même l'avoir vu. | La sueur de vos paumes sur sa peau! | Que son épaule tremble de terreur sous vos mains! | Mais j'oublie !ça ne se passe plus ainsi - | on a fait de nous du bétail du vivant de mon père ${ }^{27}$. »

Intuitive ou planifiée, la réutilisation du tuireadh gaélique dans un contexte funèbre arabe est d'un tout autre ordre que la fusion hésitante du traditionnel et du moderne dans « Le fond de l'océan ». L'étincelle du frottement culturel au Maghreb semble avoir électrifié l'imagination du poète. Une fois lancé, il rédigera 700 vers en deux mois : le récit de la jihade de l'aïeul dans les rangs d'Abd el-Kader, suivi de sa descente dans la folie et ses oraisons funèbres; et l'épopée saharienne du grand-père Omar, sans doute l'épisode le plus époustouflant du poème.

26. Pour les vers arabes, transcrits en arabe et en français dans un cahier de 1944, voir NLS MS 26729, 16-17v. Pour la lamentation de la veuve de Mokhtar, voir HAY, op. cit., p. $108-110$.

27. Comparer par exemple cet appel par la sœur d'un chef de clan assassiné avec son frère en 1663 : «Le sort du cochon noirci cramé | et de la poule sous la main du cuisinier | à tous ceux qui ont pris part au massacre! | Allez dire au Lord MacDonald | et à MacDonald des îles | que j'ai confiance devant Dieu | qu'ils poursuivront à bout la vengeance. | C'était vos cousins, c'était mes frères! " ( Cumba Ni MbicRaonaill»; voir la version publiée au Canada par A. Maclean Sinclair en 1890, dans The Gaelic Bards from 1411 to 1715, tirée des manuscrits de son aïeul : https:/ / archive.org/stream/ gaelicbardsfrom100sinc\#page/62/mode/1up (page consultée le 12 mars 2017). 
Comme Hay l'avait prédit, l'ancienne métrique orale s'adapta à merveille au mode narratif et philosophique de l'œuvre, et il l'employa pour l'intégralité du poème (hormis des pauses lyriques), jusqu'à l'épilogue "An duine agus an cogadh ${ }^{28}$ » ("L'homme et la guerre »), un lamento pour combattants décédés, qui clôt le tout :

«Saoghal fa leth mac-an-duine,

domban beò leis fhèin gach urra ;

grian is dorchadas na cruinne,

siùi-mhara 's grianstad san fhuil ann ${ }^{29}$. »

«Chaque homme est un monde en soi, | un univers entier, chaque individu ; | il a en lui le soleil et les ténèbres du monde, | dans son sang marées et solstices.

« [...] C’est un péché qui souille terre et ciel, | un jeune homme dans la saleté du combat | périssant en pleine vive-eau de la vie. «[...] C'est un crime qui aveugle soleil et astres, | les plus beaux et les plus vaillants d'entre nous | s'entretuant sans merci et se crucifiant. | Nos jeunes mis à l'épreuve - | à la fois boucher et troupeau; | les peuples de la terre par une nuit viciée | se brisant les lanternes.

«Par la chaleur torride, Mokhtar fit un geste de la main | pour essuyer la sueur, pour chasser une mouche. | Le Gefreiter tressaillit et l'obus partit.

«[... Deux univers, complexes et précieux, | furent éliminés à jamais, avant même d'avoir pu $\mid$ atteindre leur plénitude, et furent effacés | du firmament par un coup fortuit.

«Meurtre des morts, et meurtre des petits | jamais conçus - la fin de deux mondes. »

\section{Hay au Maghreb}

«J'oublie

mes sombres monts, fauves, nus,

esclave las du beau soleil ?

Or, lis

ces vers à toi tendus

du cœur splendide de l'été,

28. HAY, op. cit., p. 159-161.

29. HAY, op. cit., p. 89. 
Et dis

si tu me vois changé.

Sous les vagues de l'Algérie

la mémoire s'est-elle donc noyée?

« Je vois

ses sommets solitaires.

Leurs maitres sont en ma patrie.

L'effroi

des falaises sévères,

des rochers ascètes, surgit.

Les rois

de la Haute Kabylie

menacent impérieux, et moi,

je tiens ces fronts superbes en mépris ${ }^{30}$. »

Telles les premières strophes de «L'Écosse m'accompagne», l'un des poèmes français presque réussis qui occupent Hay dans un premier temps en Algérie. Patriotisme (ou nostalgie) oblige, car il est clair que le poète trouvait beaucoup à admirer au Maghreb, et aussi de quoi s'occuper malgré son « métier de bourriquet de bureau » et la vie « routinière, moutonnière, paperassière ${ }^{31}$ » qu'il mènera dans le calme relatif de l'après-guerre :

«L'Afrique est admirable. Il y a un air de vie et de tolérance dans les petites choses [...] qu'on ne trouve pas dans le nord-ouest de l'Europe. [...] Les gens ici font preuve de qualités qui feraient beaucoup de bien à l'Europe de l'ouest, mais l'Europe de l'ouest a toutes les mitrailleuses et se fiche bien de qualités immatérielles. Par le viseur elle ne voit rien que le phosphate, le liège, la main-d'œuvre bon marché, etc. ${ }^{32}$. »

«Tu as raison, j’ai de la chance d'être cantonné ici, avec mes connaissances des trois langues principales de la région. Parfois j'ai le sen-

30. HAY, op. cit., p. 89.

31. Lettre en français du 21 juillet 1943, NLS Acc. 6419/38a.

32. "Africa is admirable, and there is a general air of life and tolerance in small details [...] lacking in industralised N.W. Europe. [...] They show qualities here which would greatly benefit Western Europe, but W. Europe having all the machine guns doesn't worry about unmaterial things. She peers thru the sights and sees nothing beyond but phosphates, cork, cheap labour and what not. 》 (20 avril 1943) NLS Acc. 6419/38a) 
timent de m'intéresser à trop de choses, et je me dis que j'aurais beaucoup de mal à en faire la cocbur [" synthèse » en gaélique] qu'a pu faire Somhairle ${ }^{33}$. Entre les intellectuels français de Fontaine, les mystiques soufis, des bribes mal assorties de littérature italienne, les œuvres d'Anatole France, la poésie berbère, les proverbes tunisiens, et puis ce qui se fait en Écosse, en Irlande, au Pays de Galles, etc., tout ça fait un bel amalgame, et pourtant ce n'est rien par rapport à la diversité des individus que je rencontre dans la rue et sur les routes. [...] Je ne peux pas imaginer un endroit plus cosmopolite que le littoral nord-africain ${ }^{34}$.»

Son esprit semble emmagasiner tout ce qu'il lit. On discernera aisément dans sa poésie des échos de son compère Sorley Maclean ; le soufisme, les chants berbères et les proverbes arabes trouveront tous leur place dans l'épopée «Mochtàr is Dùghall» (" Mokhtar et Dou'al»). Mais l'influence de la revue mensuelle littéraire Fontaine se fait ressentir aussi, dans sa poésie et dans l'évolution de sa pensée. Fondée à Alger en 1939 par Max-Pol Fouchet, et devenue véritable tribune de la Résistance littéraire, Fontaine rassemble des auteurs vivant sous l'occupation ou en exil. On compte dans l'équipe de rédaction le penseur kabyle Jean Amrouche et le grand poète-romancier limousin Georges-Emmanuel Clancier, qui depuis l'invasion allemande de la zone sud en novembre 42 assure la liaison clandestine entre la métropole et Fouchet ${ }^{35}$. Jean Amrouche deviendra en début 44 rédacteur en chef de L'Arche, une nouvelle revue résistante sous le patronat d'André Gide, que Hay suivra également ${ }^{36}$.

33. Le poète Sorley Maclean, dans son ouvrage phare Däin do Eimbir agus dàin eile (« Poèmes à Eimhir et autres poèmes »), sorti en 1943.

34. "As you remark. I'm fortunately placed out here, knowing the three main languages of the region. Sometimes I feel I'm interested in too many things and reflect that I'd be hard put to make Sombairle's cochur of them. Between the French intellectuals of Fontaine, Süf mystics, ill-assorted odds and ends of Italian literature, the works of Anatole France, Berbrpoetry, Tunisian proverbs, and what's going on in Scotland, Ireland, Wales and so on, it's a braw mixture, yet nothing in variety to the types I meet on the streets and roads. " "I can't imagine a more cosmopolitan place than the seaboard of North Africa. » (18 avril et 3 mai 1944) NLS Acc. 6419/38a.

35. Jeanne-Marie BAUDE, Georges-Emmanuel Clancier, de la terre natale aux terres d'écritures, Limoges, PULIM, 2001, p. 36.

36. Pour ses lectures à cette époque, NLS Acc. 6419/38a (correspondance) et NLS MS 26729 (cahier de guerre). 
Dans un numéro de Fontaine, il est frappé par un douzain lyrique de G.-E. Clancier, «Le Témoin » (du groupe de poèmes « Domaine de l'homme »), décelant peut-être un ton bardique dans le mode déclamatoire du poète, porte-parole de son pays : «Je parle pour des morts qui furent bergers, [...] | Je parle pour une terre bleue très ancienne | qui bat avec mon sang, qui teinte mon regard, [...] | Je parle pour qui viendra demain jeune et dansant $[\ldots]^{37}$ ». Poussé à traduire les vers de Clancier pour un public écossais, Hay laissera de côté la mémoire des morts et en fera un simple huitain éloquent : "I speak for a blue and very ancient land | which pulses in my blood, glances blue from my eyes $[\ldots]^{38}$. 》

À un correspondant écossais (aussi poète) il fera l'éloge de la poésie de la Résistance, trouvant dans les meilleurs textes « une qualité dure et laconique très neuve en français, sans ces effets littéraires recherchés ${ }^{39}$ ». Le numéro de Fontaine où figure «Le Témoin » porte en première page le chant anonyme «Les Partisans » ("Ami, entends-tu | le noir vol des corbeaux | sur nos plaines ? [... $\left.]^{40} »\right)$, et Hay en entame une version anglaise (encore inédite) :

"Do you hear the crows, friend? | Black wings beating, sombre sound | over our plains? | Do you bear the cry, friend, | dull and muffled of our land | cast in chains?

"Ho, partisans, arm, arm! | Peasants, workers, the alarm | sounds in your ears. | The enemy tonight | will learn the price aright | of blood, with tears ${ }^{41}$.»

Un troisième texte dans ce même numéro de Fontaine, le témoignage visionnaire d'un italien en exil, influera sur le poème de guerre le plus notoire de Hay, "Bisearta » (Bizerte, voir ci-dessous). On devine aussi la lecture des courts poèmes de Jean Wahl, de sa prison à Drancy ${ }^{42}$, dans les «Stances de simple soldat » rouspéteuses que Hay esquisse en français en 43, telles que « Hypocrite » :

37. Fontaine 30 (1943), p. 499.

38. "The Witness», The New Scot (July 1947), puis Scotia Review 17 (1977), p. 49.

39. "Some of the poetry produced by the Résistance people in France is good in a way entirely new in French - laconic and hard, without any striving after those effects. » (NLS Acc. 6419/38a ; 14/2/44.)

40. «Les Partisans, chant de libération », Fontaine 30, 1943, p. 461-462.

41. NLS MS $14968,17 \mathrm{v}-18 \mathrm{v}$.

42. Jean WAHL, « Poèmes (Drancy 1941) », Fontaine 32, 1943, p. 135-150. 
Il veut passer pour exemplaire et sermonise (sic), Dieu le sait ; mais il y a bien des pas à faire de comme il faut à comme il fait ${ }^{43}$.

\section{L’homme et la lutte : " Bi iomlan is bi beò »}

En Algérie au printemps de 44, après sa "période française » (qui inclut une version dépolitisée du reproche du noyé : «Le revenant du marin parle à sa mère»), Hay se remet à composer en gaélique. «Dleasnas nan Àirdean » ("L'obligation des sommets »), un court poème déclamatoire, s'adresse à la «jeunesse de mon pays» ("ìigridh mo dhùthcha ») et appelle à la lutte sur les pics pour sécuriser le bien de la majorité dans le vallon, avant que ne frappe l'avalanche anéantissante ${ }^{44}$. L'image s'inspire sans doute du symbolisme montagneux héroïque de Sorley Maclean, mais on peut se demander si les paroles d'André Gide dans L'Arche, notées par le poète dans ses cahiers de l'époque, n'auraient pas été le point de départ du poème : "Je n'ai rien à dire aux jeunes, ... pas de conseils à leur donner, du moment qu'ils savent, préférant au repos la lutte, que rien de valable ne s'obtient jamais, de soi ni d'autrui, sans effort; que cette liberté que l'on réclame, il faut la mériter d'abord ${ }^{45}$.»

Le premier poème à thème arabe de Hay, "Athair nan Cluas » ("Père Oreilles », d'après le surnom arabe $B \hat{u} U d n i ̂ n)$, est léger : un portrait compatissant du mulet nord-africain, en route pour le marché, et courbé sous le poids des fardeaux et sous les coups de fouet de son cavalier bien en chair. Le poète loue la patience de la pauvre bête ( $B \hat{u}$ Udnîn, ces oreilles | qui sont l'insigne de ta tribu, | ne sont-elles pas tout aussi longues que la patience | qu'Allah te légua comme marque de beauté ? ») ; mais il admire aussi la graine d'insoumission dans son caractère. Le poème charme par sa simplicité et sa chaleur, mais s'inscrit dans une lignée de poèmes datant de la période maghrébine et menée à bout en Italie, traitant de thématiques philosophiques et sociales. «Père

43. HAY, op. cit., p. 91. Comparer par exemple « Desperado » de Wahl : « La vie est là simple et tranquille; | séparés d'elle par un mur | nous regardons tous immobiles | ce peu de vert, ce peu d'azur. »

44. HAY, op. cit., p. 102.

45. NLS MS 26729, 12r. A. GIDE, « Appel», L'Arche 1, 1944, p. 13-15, p. 15. 
Oreilles » est en quelque sorte un premier essai pour le grand poème «Atman » écrit en quatrains très similaires, qui fait l'éloge d'un Algérien pauvre de Dréan (Mondovi à l'époque), incarcéré sans pitié pour une infraction mineure :

« Tu volas par nécessité, | tu mentis pour t'en tirer; | tu fus condamné, châtié et fouetté, | et ils te mirent sous verrou.

«La bouche honorable qui te condamna | était petite et grasse dans un visage grisâtre ; | la Justice avait les yeux chassieux, à tant examiner | son abondance de livres comptables.

"Mais la bouche jugée mensongère | était courtoise, enjouée, mélodieuse ; | j’en tirais des histoires et de la bonne repartie, | bien qu'elle ne connût pas trop les repas.

«Tu levais le regard de ton travail | pour tirer plaisir du monde alentour. | Tu me faisais l'éloge du Djebel Iussuf, | ses couleurs et ses contours.

«Je te connais, Atman : | la femme de ton foyer et tes cinq petits, | ta poignée de chèvres et ton mulet, | ton petit terrain de seigle, et ta vache.

"Je te reconnais, Atman : | tu es homme, et tu es vivant; | deux qualités dont le juge est dépourvu | et qui lui sont perdues à jamais.

«Ton front est souvent en sueur, | tu connais l'allégresse et la colère, | tu as goûté et regoûté l'écart | entre le doux et l'amer.

«Tu as connu la haine, la joie, le chagrin, | tu as connu la tempête et le soleil ; | tu as vécu la vie à fond | et tu n'as jamais fléchi devant elle.

«Si tu étais riche, et ta tripe | engraissée par la maigreur de tes laboureurs éreintés, | tu ne serais pas à Mondovi à partager | ton sombre cachot avec les poux.

"Autant je tournerai le dos | à l'honorable magistrat, | je traverserai la rue | pour te saluer si je t’aperçois.

«Sîdna Aïssa fut crucifié | sur une colline entre deux voleurs, | et ce serait blasphème, Atman, de nier | que pour moi tu es un frère. »

Si dans «Père Oreilles » l'appellation Bû Udnîn en plein poème créé un petit choc linguistique, pour le lectorat gaélophone l'allusion au Christ par son qualificatif arabo-musulman dans « Atman » est bien plus incongrue (même si elle ne porte pas l'athéisme affiché de Sorley 
Maclean ${ }^{46}$ ); la moralité calviniste bien-pensante de l'époque pouvait trouver également déplacé l'éloge du voleur. Pourtant la fraternité entre fellah algérien et crofter (petit agriculteur) des Highlands est évidente, tous deux enracinés dans une riche culture orale malgré leur extrême pauvreté. Il est pertinent de noter que, dans un cahier de l'époque, Hay transcrit des propos d'un article paru dans Alger Républicain de mai 44 (quelques mois donc avant la rédaction finale d'« Atman ») sur l'exploitation des ouvriers agricoles, dont les qualificatifs dénigrants cités rappellent mot pour mot le portrait si souvent dressé des Highlanders gaélophones depuis le XVIII siècle :

«Je connais le vieux portrait des indigènes Algériens, tel que le brossent certains : sales, imprévoyants paresseux menteurs voleurs - le portrait que brossent les responsables de cette misère. Ils sont devenus fellahs, sans terre, ou il ne leur est resté que des terres pauvres, ingrates, et si étroites qu'en ces climats de dur soleil elles ne peuvent pas nourrir leur famille ${ }^{47}$. »

En dépit de la prison et de la pauvreté, Atman est pleinement «homme», riche en vécu humain, vivant la vie à fond, enraciné, et généreux dans son partage culturel. Les forces sociales qui empêchent le plein vécu et le plein partage - les conventions entravantes et les opinions d'autrui - font le sujet d'un poème de la même période, "Priosan da fhèin an duine ${ }^{48}$ ? 》 ("L'homme sa propre prison ? »). Aux images bien écossaises ("Vois le fou de Bassan plonger comme une flèche », «le vif tartan de [notre] nature délavé à un gris indifférencié »), le poème vise peut-être le calvinisme contraignant et ascète des Highlands, mais plus généralement la déculturisation de la société gaélique. Avec un optimisme et une foi dans la nature humaine qui fait l'antithèse de ses propos sur la guerre, il exhorte l'individu à la libre affirmation de différence : «cœur et esprit ne te tromperont pas, | sois pleinement toi et vis ta vie ... Vis à fond et sois toi-même » ("Cha chuir ceann is cridh" air iomrall thu, | bi iomlan is bi beò... Bi beò is bi thu fhèin »).

46. Voir «Ban-Ghäidheal» («Femme des Highlands») ou «An crann dubh » («L'arbre noir »), dans S. MACGill-Eain, O Choille gu Bearradh Collected Poems, Manchester \& Edinburgh, Carcanet/Birlinn, 1999, p. 26-28, p. 36.

47. NLS MS 26729, 19r.

48. HAY, op. cit., p. 174-176. 
L'appel à assumer sa différence est lancé non pas à un individu mais à un peuple dans le long poème gaélique à titre arabe "Meftah Bâbkum es-Sabar ${ }^{49}$ ? " ("La patience, la clé de ton cachot?»). Sur un fond tunisien, en paragraphes monorimes (la métrique du lamento), il reprend le trope de l'emprisonnement à travers l'aphorisme soufi du titre, pour remettre en question l'idée même du fatalisme.
«Is cuimhne leam an Sùg el-Cheamais
sa chaifich dhorcha is sinn a' deasbad,
guth cianail mar ghuth chlag fo fheasgar
a mhol domh striochdadh don Fhreastal.»
"Je me souviens une fois à Souk el-Khemis, nous discutions dans l'obscurité du café,
et une voix mélancolique comme les cloches du soir
me conseilla de me soumettre à la Providence. »

Le poète répond que "pour nous un choix à part est écrit : | la paix et la mort, ou la lutte et la vie » ("sgriobhadh roghainn fa leth dhuinn, | an t-sith 's am bàs, no gleac 's a' bheatha »). Malgré les expulsions (des HautesTerres) et les dépeuplements, "nous sommes encore là, | et sous les cendres brûle encore la braise ». La suite du poème est un plaidoyer au lectorat de ne pas détourner ses poètes de leur devoir, de ne pas vouloir d'eux des poncifs nostalgiques, des idées reçues, des berceuses ou des romances, de la féérie ou "l'ensorcellement mélodieux de locutions raffinées » ("draoidheachd cheòlmbor fhacal liombta »), mais plutôt : «Exigez rires, bonne humeur et colère, | amitié et inimitié, plaisir et déplaisir - | Exigez le vrai miroir de notre pensée. | Cherchez dans ce que nous créons | la vie rude, douloureuse, exultante, | car la Providence nous a offert en nos jours | le choix entre la vie ou la mort. »

Si l'iconographie de la soumission dans le poème est avant tout arabe, il est clair que le poète renie un fatalisme bien écossais (calviniste ou autre) qui menace de paralyser la culture du pays et «délaver le tartan vif » par une passivité politique et culturelle à racine historique (qu'il dénoncera dans un poème anglais comme «faux récit visant à estropier l'esprit, retenir la main », «noir mensonge de notre pénurie 
prédestinée $\left.{ }^{50} »\right)$. En conclusion de «Meftah», le poète évoque le renouveau culturel que pourrait engendrer une volonté créatrice collective tournée vers l'avenir mais s'inspirant du passé :

« le champ qu'éveilleront nos attelages; [...] | la salle de fête que nous découvrîmes morne | mais qui s'emplira matin et soir | de nos chants et de la musique de nos ancêtres ; | le livre où nous inscrirons | de nouveaux vers sous la dernière strophe | qu'y laissèrent les poètes de jadis - | tel sera notre pays. Ou alors, s'il n'y a pas lutte, | une chose insignifiante cachée dans un recoin, | qu'un autre peuple saigna et oublia ».

Le fil existentialiste qui court par ces poèmes (et d'autres de l'époque) et les questions qui y préoccupent leur auteur - sur ce qui fait l'« homme », le vécu intégré, l'affirmation et le partage culturel, le devoir du poète et les liens entre poète et peuple - doivent beaucoup à l'environnement intellectuel dans lequel Hay se trouva plongé (ou se plongea) durant les années de guerre. Les apports de la littérature francophone en particulier (mais aussi italienne) sont mis en évidence dans un long essai rédigé en 1944 et publié après la guerre, "Poetry in the world or out of it? " ("La poésie au cœur du monde ou en dehors?»), ou Hay discute l'hermétisme et les « tours d'ivoires » littéraires, le droit de toute classe sociale à l'art, et le devoir du poète envers ses prochains. Il prend parti contre l'élitisme exprimé par André Gide dans Les Lettres Françaises auquel il préfère l'idéalisme démocrate de Benedetto Croce (qu'il aura rencontré dans L'Arche) ainsi que l'engagement des intellectuels de Fontaine parmi d'autres ${ }^{51}$ :

«La poésie n'est pas faite par des jongleurs de mots, mais par des hommes qui ont un cœur, un esprit, un corps, qui parlent aux hommes et parlent pour les hommes. Quand ils parlent, leurs paroles viennent du cœur de la vie, comme Garcilaso de la Vega [sol-

50. «False tale to lame the spirit, hold the hand. | ... black lie of our predestined dearth» ("The Walls of Balclutha », 1946), HAY, op. cit., p. 233. Dans la nouvelle gaélique "Le Grand Café de la Jeunesse, 1942 », un soldat des Highlands rappelle à son compagnon que « si [au Maghreb] les gens ont tendance à se plier aux circonstances, ... nous avons nous aussi notre dicton: Ce qui est destiné adviendra. » HAY, op. cit., p. 597.

51. G. C. HAY, « Poetry in the world or out of it », «1. André Gide and the ineffable », Scottish Art and Letters 2, 1946, p. 49-58; «2. The time, the money or the inclination », Scottish Art and Letters 3, 1947, p. 52-57. 
dat poète, $\mathrm{XVI}^{\mathrm{e}}$ siècle] et les poètes français de la Résistance, et non pas du dehors de la vie.

[...] Malgré l'histoire de ce siècle, ensanglanté comme aucun autre, de toutes parts des écrivains proclament [...] qu'on peut tout espérer de l'homme. [...] Comme le déclare la préface de l'anthologie [résistante] L’honneur des poètes [...] : "Il est temps de le dire et de le proclamer : les poètes sont des hommes comme les autres [...]". [...] Louis Julia dans Afrique Littéraire n'hésite pas : "Nous écrivons, non pas pour nous, mais pour les autres. [...] Les peuples ont droit à l'art comme ils ont droit au pain". »

Et pour évoquer les poètes qui atteignent l'universalisme à travers leur appartenance locale, Hay citera côte à côte Hugh MacDiarmid et Georges-Emmanuel Clancier, puis Jean Amrouche, «cet écrivain accompli de souche berbère qui dit que la poésie est le langage de l'être total, ou, comme je l'ai exprimé, de l'être intégré ${ }^{52}$ ».

\section{Poésies de guerre}

L’optimisme exprimé par Hay dans ses poèmes sur thème d'action et de volonté créatrice est réduit au silence dans ses poèmes de guerre. Spectateur-chroniqueur plutôt que combattant actif et impliqué, il se porte témoin du chaos et de l'effondrement de la société civile. «Truaighe na h-Eòrpa ${ }^{53}$ » («La misère de l'Europe »), rédigé en Grèce, est une courte lamentation en distiques déclamatoires pour un continent en ruine et une marée humaine en déroute :

«Tha mùir shnaidhte na b-Eòrpa

shios nan tòr air a raointean.

"Tha an gràbhaladh àrsaidh

air a sgaineadh is gaorr air. »

«Les remparts gravés de l'Europe | gisent en débris sur ses plaines. «Leurs sculptures anciennes, | éclatées et souillées de sang. »

Le poète plaint le sort des réfugiés de guerre, dont «les cris de

52. Voir Jean Amrouche, "Pour une poésie africaine », Fontaine 30, 1943, p. 531-543, p. 536. Pour l'anglais, voir HAY, art. cit. (1), p. 52 et 57.

53. HAY, op. cit., p. 215. 
misère aigus percent | au-dessus des vents mugissants» ("Chaidh geurghuth an truaighe | thar cruaidhgair a gaothan »), et s'exclame avec un eurocentrisme désespéré que le continent, «le vieux sanctuaire des arts, | le tendre cœur de l'humanisme », n'est plus qu'un « cap de l'Asie, les Balkans du monde » ("Och, Rudha na h-Ásia, | Bälcan an t-saoghail! »). Pour le lecteur gaélophone le titre du poème, «La misère de l'Europe», ne peut manquer de rappeler une des poésies lyriques célèbres du confrère Sorley Maclean ${ }^{54}$, mais le lecteur français entendra peut-être dans ses premiers distiques «l'Europe aux anciens parapets » de Rimbaud, et y devinera le filet des lectures maghrébines du poète ${ }^{55}$.

Hay dépeint encore l'anéantissement de la civilisation humaniste dans son seul poème anglais de la guerre, «Esta Selva Selvaggia " ("Cette forêt sauvage ", citation de l'Enfer de Dante), un collage en tercets dantesques presque cinématographique de scènes et de conversations brutales, tragiques, désespérées, allant de l'Écosse jusqu'au Maghreb, l'Italie et la Grèce, où l'on retrouve la qualité claire, " dure et laconique », que Hay admirait dans la poésie de la Résistance de Fontaine. Le bombardement de Clydebank (aux environs de Glasgow, en 1941) évoqué au tout début, unit « enfin » l'Écosse à l'Europe dans une « civilisation commune » du chaos : « sirènes, explosions, désintégration » («At last we found a civilisation | common to Europe and our nation, | sirens, blast, disintegration »). La fresque implacable atteint son point culminant dans un baragouin plurilingue de désespoir, d'auto-disculpation et de dénonciation haineuse entre peuples, suivi d'un avertissement du soldat narrateur : «Listen to yourselves. Beware ${ }^{56}$. » («Écoutez-vous. Prenez garde. »)

54. «Gaoir na h-Eòrpa » (« Le cri d'agonie de l'Europe ») de 1938, bien que cette locution inoubliable de la première strophe ne sera reprise comme titre que beaucoup plus tard. Voir MACGILL-EAIN, op. cit., p. 8-9.

55. Le vers de Rimbaud est cité très visiblement (adapté à « retrouver l'Europe aux anciens parapets ») dans un article de H. NICOLSON, «La nouvelle entente cordiale », Fontaine 33 (1944), 336. Voir « Le bateau ivre », A. Rimbaud, CEuvres complètes : poésie, prose et correspondance, Libraririe Générale Française, 1999, 297.

56. HAY, op. cit., p. 211-214. Pendant longtemps très peu connu, « Esta selva selvaggia » figure dans une récente anthologie des deux guerres, dont les éditeurs estiment qu'il doit compter parmi les plus grands poèmes de guerre anglophones : «Written in English of an almost unbearable intensity, this must be one of the great war poems of the age », D. GOLDIE, R. WATSON (éd.), From the line : Scottish war poetry 1914-1945, Glasgow, ASLS, 2014, xviii. 
«Bisearta ${ }^{57} »\left(«\right.$ Bizerte »), le poème le plus acclamé de Hay ${ }^{58}$, situera également les origines du conflit mondial dans le cœur humain plutôt que sur le plan géopolitique. Le poème, qui évoque l'horreur du bombardement de Bizerte dans la dernière poussée de la campagne de Tunisie au printemps 43, trouvera sa forme aboutie deux ans plus tard en Italie. L'image envoûtante du feu - une bête ailée, à la fois épouvantable et magnifique, dont le mouvement hypnotique domine le poème - doit sans doute au chef-d'œuvre du père de Hay, le roman Gillespie (1913). Dans un des grands tableaux du roman, le feu qui va dévaster la flottille de pêche a « un mouvement rythmique qui fascinait l'œil [...]. Comme la tête d'un serpent [il] avançait et reculait en oscillations lentes [... et] hypnotisait par sa terrifiante splendeur ${ }^{59} »$. Mariée au terrible magnétisme de l'image, la pulsation rythmique de «Bizerte» est sans antécédent dans la littérature gaélique: elle est dérivée d'une métrique pétrarquienne que Hay rencontre au cours de ses lectures en $44^{60}$, et qu'il greffe à sa propre tradition par un jeu d'assonance au sein de chaque distique.

Bisearta (Bizerte)

«J'aperçois, durant la garde de nuit, un sinistre frémissant qui ourle l'horizon.

Battant des ailes, il dissipe et disperse là-bas les astres du ciel.

«On croirait que se feraient entendre du cœur du feu si loin qu'il soit, pleurs et lamentations, rugissements furieux et cris de haine, le glapissement des chiens enragés ou le hurlement des loups, on croirait que le grondement sourd de sa violence

57. HAY, op. cit., 176-177.

58. «Perhaps the finest poem of total war », GOLDIE, WATSON, ibidem, xvii-xviii. Le poème fut traduit en scots par Hugh MacDiarmid peu de temps après sa rédaction, pour l'anthologie phare de la Renaissance, Modern Scottish Poetry 1925-1945 éd. M. Lindsay (London, Faber, 1946 ; rééditée en 1966, 1976 et 1986), p. 78-79.

59. "It had a rhythmic movement which fascinated the eye. [It] oscillated backwards and forwards slowly, like the head of a snake [and its] splendour and terror mesmerised ", J. M. HAY, Gillespie, Edinburgh, Canongate, 1979 [1913], p. 253.

60. Dans un cantique attribué au prêcheur Savonarole, $15^{\mathrm{e}}$ siècle. Voir G. C. HAY, op. cit. p. 593. 
atteindrait, de cette fournaise ambrée, la limite extrême du monde ; mais voilà qu'il se répand au loin, le long de l'horizon, dans un silence effroyable, maléfique.

«Quels sont leurs noms, ce soir, les ruelles miséreuses où de chaque fenêtre jaillissent flammes et fumée et étincelles, et les cris de terreur des malheureux dont les maisons étripées s'abattent dans un tourbillon de fumée? Qui donc, ce soir, supplient la Mort de venir vite, en toutes leurs langues, ou se débattent parmi pierres et poutres, criant au secours, fous de panique, mais que nul n'entend? Qui ce soir est en train de payer la vieille taxe accoutumée du sang populaire?

«Par moments rouge comme une mare sur le champ de bataille, puis blême comme la pâleur fétide de la peur, grimpant et retombant, se lançant d'un bond soudain et se rapetissant, perdant ses forces un moment puis se gonflant comme le souffle d'un diable en furie, je vois devant moi le Mal, tel un cœur et un pouls, dont les pulsations baissent et surgissent. Le feu, cette horreur à l'horizon, cet anneau d'or et de rose au creux de la nuit, renie et démentit par sa lueur la tranquillité antique et sublime des astres. »

Connaissant les capacités éclectiques étonnantes de Hay, il est intéressant de noter un troisième texte (après Gillespie et Savonarole) qui, emmagasiné par Hay au cours de ses lectures maghrébines, aurait pu contribuer à l'éclosion de son plus éloquent poème. «L'Incendie » est un émouvant appel à l'espoir par l'exilé antifasciste Enrico Terracini, traduit en français par sa femme (la romancière Jeanne Terracini), et publié dans le numéro de Fontaine dont il a déjà été question ci-dessus (voir « Hay au Maghreb »). L'écrivain dissident s'unit, par l'imagination et la sensibilité, à la souffrance de ses compatriotes « frères, compagnons, gens du peuple » : 
« De vos pieds courant dans la nuit haute, épaisse d'ombre, j’entends la fuite frénétique ... et je guette autour de moi, perdu dans les ténèbres, haletant du désir d'être avec vous..., pour vous parler cœur à cœur, pour penser avec vous que de l'Apocalypse biblique d'aujourd'hui, il ne restera rien, pas même les cendres.

« De même que je suis contre vous quand vous oubliez votre humanité, je suis avec vous, frères, ... quand les cris des enfants se répercutent contre le ciel comme contre une tombe, quand les mères s'abandonnent aux imprécations, quand les vieux se plaignent et les hommes se taisent.

« [...] Il y a l'incendie chez vous, l'incendie de l'Italie [...] C'est l'incendie de notre terre et je le sens, je discerne les flammes, comme si l'espace n'était plus entre vous et moi, comme si le regard saisissait le brasier atroce des pins maritimes qui s'élèvent sur les pentes rapides, au-dessus de la plage $[. .$.$] - un cri de vert mêlé à l'or$ du feu au-dessus de l'écume de la mer.

« [...] Et voilà que cela a été, l'incendie brûlant et dur, rougeâtre à l'ombre des blanches églises et des marbres striés, sombre dans les venelles et les rues étroites, crépitant sous les toits, et encore féroce aux dernières étoiles du matin.

«Mais je ne peux croire ni ne me sens capable d'imaginer. Et mes mains qui ont travaillé dans les ports du monde, je les porte à mes yeux pour ne plus distinguer vos visages aux premières lueurs de l'aube. Et ces mêmes mains je les porte à mes oreilles pour ne plus entendre vos cris ${ }^{61}$.»

Face à l'apocalypse, Terracini rêve d'une aube d'espoir et ose espérer que la flamme de la liberté succédera aux feux de la guerre dans son pays ${ }^{62}$. Quand le poète écossais nous plonge au cœur de la terreur de Bizerte, les victimes restent anonymes (et également leurs bourreaux), piégés dirait-on dans l'étau de l'histoire, condamnées au sort de toutes les petites gens à travers les siècles. Dans «Bizerte», aucune parole d'espoir ou cri de liberté ne retentit dans l'impassible beauté des astres, et dans aucune de ses méditations sur la guerre Hay ne saura évoquer une vision rédemptrice qui justifierait le cataclysme. Comme exprimé en épilogue à « Mochtàr is Dùghall» (voir «Un modernisme prudent», ci-

61. E. Terracini, «L'incendie », Fontaine 30, 1943, p. 481-485, p. 481 et 483.

62. TERRACINI, op. cit., p. 484-485. 
dessus), la guerre est avant tout et toujours « un péché qui souille terre et ciel, | [...] un crime qui aveugle soleil et astres ».

\section{Rencontres culturelles : « Mokhtar et Dou’al »}

L'essor que prirent la pensée et l'imagination de Hay en Afrique trouva son expression la plus étonnante dans son chef-d'œuvre inachevé et longtemps inconnu, «Mochtàr is Dìghall » ("Mokhtar et Dou'al»), fresque narrative et philosophique de presque 1300 vers, ponctuée de courts poèmes lyriques ${ }^{63}$. Sans trop s'attarder sur ce qui est un grand poème de guerre (car dans chaque épisode les conflits violents sont au premier plan ou servent de fond), notons que le poème est aussi un hommage aux sociétés orales en voie de disparition et un hymne à la diversité humaine et la différence culturelle. Par le biais des aïeuls de Mokhtar (dont la mémoire est transmise par la culture orale de la famille) Hay célèbre les valeurs à échelle humaine d'une société prétechnologique, et recrée non seulement une culture mais toute une gamme d'humanité (masculine) : l'arrière-grand-père Ahmed, guerrier sous Abd el-Kader ; le grand-père Omar, marchand-aventurier bon vivant et grand conteur ; et son fils Obaï, mystique ascète mort jeune.

On retrouve certains rapprochements dans le poème entre l'ancienne société gaélique et la société (traditionnelle) arabe, et une équivalence est tout au moins suggérée dans le cadre de la colonisation (même si Hay n'ignore pas le rôle colonisateur des Écossais au sein de l'armée britannique); mais la question des différences culturelles et (socio)linguistiques est approfondie de manière plus subtile dans l'épopée d'Omar au Sahara. En effet Hay fait chavirer la perspective de son lectorat gaélophone en dépeignant la tribu touareg (qui assiège la caravane d'Omar) uniquement par le biais de son conteur arabe, à la fois terrifié et méprisant de cette culture subalterne. Les propos d'Omar sur l'apparence, les coutumes et la langue des barbares (berbères) ne peuvent manquer d'évoquer la méfiance et le mépris dans lequel la so-

63. On reconnait, dans la structure et l'ambition du poème, l'influence du long poème épique socialiste de son compère Sorley Maclean «An Cuilithionn » ( Le Coolin » de 1940, nom de la chaîne montagneuse qui domine l'île de Skye). À noter que Maclean et Hay furent tous les deux inspirés par le chef d'œuvre de Hugh MacDiarmid « $A$ drunk man looks at the thistle » ("Un ivrogne contemple le chardon ») de 1926. 
ciété anglo-scotophone a longtemps tenu la culture gaélophone (dont les clans avaient aussi l'amour du dithyrambe guerrier, et leur ancienne coutume de la creach, tout comme la razzia targuie dont Omar est victime). Par surcroît, quand Omar décrit le parler touareg comme un « gloussement», un « bégaiement », " pâteux, étranglé, cassé, rauque », « comme si l'homme souffrait d'une luette enflée qui l'étouffait ${ }^{64}$ », on croirait entendre (en gaélique!) une description de l'arabe même par un Roumi ignare. C'est avec un humour rare, et en renversant le poncif colonialiste du pirate du désert, que Hay fait réfléchir sur le relativisme culturel et l'aspect sociopolitique des rencontres entre peuples ${ }^{65}$.

«Mokhtar et Dou'al» reste inachevé dans son volet écossais, mais la grandeur du volet arabe a suffi pour gagner la louange des critiques. Pour Ronald Black, éditeur de l'anthologie-clé de la poésie gaélique du XXe siècle, « la poésie nord-africaine de Hay, et en particulier [...] Mochtàr is Dùghall, a élargi le champ de la poésie gaélique plus encore que la psychanalyse littéraire dans laquelle s'est aventuré [le grand moderniste Sorley] Maclean ${ }^{66}$. » Et encore :

« On $[y]$ trouve profondeur philosophique, musique, humour, ambiance, couleur, suspense, et une richesse conceptuelle qui coupe le souffle, et on pourrait conclure que tout compte fait [...] Hay trouva son âme en Algérie et que ce grand poème inégal démontre bien qu'il était moins barde de Kintyre que barde d'Afrique ${ }^{67}$.

64. HaY, op. cit. p. 127.

65. Pour une étude de ces questions vis-à-vis des convictions politiques de Hay, voir C. WHYTE, "George Campbell Hay: nationalism with a difference », dans D. S. Thomson (dir.), Gaelic and Scots in Harmony, Glasgow, Dept of Celtic, University of Glasgow, 1990, p. 116-135.

66. «Hay's North African verse, and especially [...] Mochtàr is Dùghall, broadened the range of Gaelic verse still further than did MacLean's ventures into psychoanalysis.» R. BLACK, «Twentieth century Gaelic poetry », dans S. MacMathúna , A. Ó Corráin (dir.), Celtic Literatures in the Twentieth Century Moscow, 2007, p. 49-96, p. 67.

67. «It has philosophical depth, music, humour, atmosphere, colour, excitement, and a conceptual richness that takes the breath away. [...] It may be suggested, all in all, that [...] Hay found his soul in Algeria, and that this great but lopsided poem shows us that Hay was not so much a Bard of Kintyre as a Bard of Africa. » R. I. M. BLACK, An Tuil: anthology of twentieth century Scottish Gaelic poetry, Edinburgh, EUP, 1999, xxxviii. C'est l'historien-poète Angus Martin qui dénomme Hay « Bard of Kintyre » dans sa biographie du poète ; voir MARTIN, op. cit., p. 48 . 


\section{Conclusion}

Les écrits «maghrébins» de Hay (ou rédigés au Maghreb, ou inspirés par la région et par ses lectures de l'époque) s'inscrivent dans une démarche culturelle d'ouverture et de découverte de l'autre très marquée. Les transferts et les emprunts linguistiques et littéraires sont au cœur de son œuvre, pour des raisons d'intérêt et de curiosité personnels bien sûr, mais aussi par service à la culture (ou aux cultures) de son pays, afin d'assurer des passerelles civilisatrices entre l'Écosse et le monde, à une époque où l'effondrement social et le barbarisme menaçaient. On peut aussi proposer, comme l'a fait une critique (luimême poète, et traducteur plurilingue), que cette démarche trouve une de ses sources dans le creuset identitaire de son enfance :

«En faisant le choix d'écrire en gaélique, une langue parlée ni par ses parents ni dans son propre milieu social, Hay avait lui-même franchi une limite culturelle. C'est peut-être cette expérience d'être devenu "autre" (ou de se faire passer pour "autre") qui l'encouragea à faire un saut vers d'autres "autres". Son engouement pour la culture arabe est l'expression la plus saillante de ce phénomène ${ }^{68}$. »

Les nombreuses lectures françaises (et par la suite italiennes) de Hay le conduisirent à réfléchir sur des questions culturelles et existentielles pertinentes à l'Écosse en les situant dans un champ intellectuel de plus large empan. Les textes qu'il découvrit dans des revues telles que Fontaine influèrent, directement ou plus souvent de manière oblique, sur sa propre création littéraire. Un enraciné mordu de la diversité, il franchit avec enthousiasme et imagination les limites linguistiques et culturelles qu'il rencontra au Maghreb (et ailleurs), et ce faisant créa un nouvel espace dans la littérature gaélique ouvert à l'Europe, le monde arabe et le monde non-anglophone.

68. "By choosing to write in Gaelic, in a language used neither by his parents nor in his own social ambience, Hay had himself crossed a cultural boundary. The experience of becoming or impersonating an "other" may well have encouraged a leap in the direction of other "others". His fascination with Arab culture is the most prominent instance of this phenomenon. »C. WHYTE, Modern Scottish Poetry, Edinburgh, EUP, 2004, p. 88. 


\section{Textes gaéliques :}

\section{Atman}

Rinn thu goid nad èiginn,

Dh'fheuch thu breug gu faotainn às;

dhit iad, chàin is chuip iad thu, is chuir iad thu fo ghlais.

Bha 'm beul onorach a dhit thu pladach, bìdeach sa ghnùis ghlais;

bha Ceartas sreamshùileach o sgrùdadh

a leabhar cunntais 's iad sior phailt.

Ach am beul a dhearbhadh breugach, bha e modhail, èibhinn, binn;

fhuair mi eirmseachd is sgeòil uaith

's gun e ro eòlach air tràth bìdh.

Thogte do shùil on obair

à cruth an t-saoghail a dheoghal tlachd;

mhol thu Debel Iussuf dhomh, a cumadh is a dath.

Is aithne dhomh thu, Atmain, bean do thaighe 's do chòignear òg, do bhaidnein ghobhar is d' asail, do ghoirtein seagail is do bhò.

Is aithne dhomh thu, Atmain: is fear thu's tha thu beò, dà nì nach eil am breitheamh, 's a chaill e 'chothrom gu bhith fòs.

Chan ainmig d' fhallas na do shùilean; is eòl duit sùgradh agus fearg; bhlais is bhlais thu 'n difir eadar milis agus searbh. 
Dh'fheuch thu gràin is bròn is gàire;

Dh'fheuch thu ànradh agus grian;

Dh'fhairich thu a' bheatha

is cha do mheath thu roimpe riamh.

Nan robh thu beairteach, is do chaolan

garbh le caoile d' airein sgith, cha bhiodh tu 'chuideachd air na miolan

an dubh phrìosan Mhondovì.

Nuair gheibh breitheamh còir na cùirte

làn a shùla de mo dhruim, thig mi a thaobh gu d' fhàilteachadh trasd an $\mathrm{t}$-sràid ma chì mi thu.

Sidna Aissa, chaidh a cheusadh mar ri mèirlich air bàrr slèibh, is b' e 'n toibheum, Atmain, àicheadh gur bràthair dhomh thu fhèin.

\section{Bisearta}

Chì mi rè geàrd na h-oidhche dreòs air chrith na fhroidhneas thall air fàire, a' clapail le a sgiathaibh, a' sgapadh 's a' ciaradh rionnagan na h-àird' ud.

Shaoileadh tu gun cluinnte, ge cian, o 'bhuillsgein ochanaich no caoineadh, ràn corraich no gàir fuatha, comhart chon cuthaich uaith no ulfhairt fhaolchon, gun ruigeadh drannd an fhòirneirt on fhùirneis òmair iomall fhèin an t-saoghail. Ach siud a' dol an leud e ri oir an speur an tostachd olc is aognaidh. 
C' ainm nochd a th' orra,

na sràidean bochda anns an sgeith gach uinneag

a lasraichean's a deatach,

a sradagan is sgreadail a luchd thuinidh, is taigh air thaigh ga reubadh, am broinn a chèile am brùchdadh toit a' tuiteam?

Is cò a-nochd tha' $\mathrm{g}$ atach

am Bàs a theachd gu grad nan cainntibh uile, no a' spàirn measg chlach is shailthean

air bhàinidh a' gairm air cobhair, is nach cluinnear?

Cò a-nochd a phàigheas

seann chìs àbhaisteach na fala cumant?

Uair dearg mar lod na h-àraich, uair bàn mar ghile thràighte an eagail èitigh, a' dìreadh 's uair a' teàrnadh, a' sìneadh le sitheadh àrd 's a' call a mheudachd, a' fannachadh car aitil 's ag at mar anail dhiabhail air dhèinead, an t-Olc na chridhe 's na chuisle, chì mi na bhuillean a' sìoladh a' leum e. Tha 'n dreòs na oillt air fàire, na fhàinne ròis is òir am bun nan speuran, a' breugnachadh 's ag àicheadh le shoillse sèimhe àrsaidh àrd nan reultan.

L'auteur tient à exprimer sa gratitude envers le CRBC et en particulier Philippe Jarnoux, alors son directeur, pour l'avoir accueilli comme professeur invité en avril-mai 2016 ; et tient également à remercier Mannaig Thomas et Nelly Blanchard de l'avoir invité à participer au séminaire La Bretagne linguistique du 29 avril 2016. 